\title{
Forces attributed to dark matter may originate from entangled particles as seen in the shape of galaxies formed by GRBs
}

\author{
S. BUGLYó \\ University of Debrecen, buglyos@gmail.com
}

Abstract. Recently, it has been suggested that entangled particles may be connected by a wormhole. If that is right, what is the distance between them we have to take into account when applying Newton's law of universal gravitation to these particles? We propose the idea that these particles may attract each other regardless of distance, resulting in a force that behaves exactly the same way as the force derived from presumed dark matter. Traces of such a force seem to be present in galaxies due to gamma ray bursts (GRBs) that produce entangled particles which hit various objects. We can observe that in barred spiral galaxies the arms always pass through the nucleus of the galaxy so we believe that the very first GRB happened at the central supermassive black hole (SMBH) and the arms are the traces of this ancient GRB. If we see an unbarred spiral galaxy, we can be certain that the arms do not pass through the core and we think the very first GRB happened close to the core. Ring galaxies may also be considered as a type of spiral galaxies, since there is a section where the ring is broken, i.e. where the arms do not meet. So the very first GRB happened far from the core. Elliptical galaxies may have resulted from an ancient GRB which hit from outside. The arms rotating in opposite directions of the NGC4622 galaxy support our hypothesis. Finally in the silk threads of the spider web of the universe, the traces of GRBs can be seen.

\section{Introduction}

Recent findings reported in the literature have called the existence of dark matter into question (Müller 0., et al. 2018). We aim to explain the structure of the universe and the formation of the various types of galaxies. It is well known that if a measurement is conducted on one particle of an entangled pair, it is possible to determine the spin and other physical parameters of the other particle from large distances (Horodecki R., et al. 2009, Einstein A., et al. 1935). To explain the phenomenon, the particles have been proposed to be connected by a wormhole (Maldacena J. \& Susskind L., 2013, Grant A., et al. 2015). In such a case, we can reasonably assume that entangled pairs of particles with a non-zero mass attract each other regardless of distance. Their distance through the wormhole is almost zero, so we must take the gravitational attraction between the particles into account.

Let us now examine the consequences of this assumption in theory:

- This would bring about no change in the Solar System, as the gravitational forces are considerably greater due to the relatively shorter distances between the planets, masking the effects caused by such a small force of attraction.

- Its effect, however, would be detectable in a galaxy and the universe, due to the large distances. 


\section{Effect on the formation of galaxies}

Particles of an entangled pair colliding with two different objects is probably a rare phenomenon in nature. For example, entangled pairs of photons that arise from the Sun's radiation probably travel together and impact together into the same object. It seems likely that in order to have entangled particle pairs impact into different objects, powerful events of the universe are required, such as the engulfment of a star by a black hole or the collision of two black holes, or perhaps quasars and pulsars may release such particle pairs. In such cases, a huge number of entangled pairs of particles fly across the space to hit different objects, and their effect on the movement of stars may not be negligible. What we see as the most probable case is that entangled particles may be generated in masses during gamma-ray bursts (GRB), where a whole star's material is emitted in a jet. So the objects hit by this jet will attract one another in pairs. We think that gamma-ray bursts and the peak intensities of their light curves (Mészáros P. \& Rees, M. J. et al. 1997, Costa E. et al. 1997) are responsible for the various shapes of galaxies, as described below in detail. (Toth G. \& Ostriker J. P. 1992, Block D. L. \& Wainscoat R. J. 1991) Then, applying our hypotheses, we will try to unravel how the various types of galaxies might have evolved. Let us consider a young galaxy, similar in appearance to an elliptical galaxy from the outside: it has a supermassive black hole in the centre, surrounded by stars on elliptical orbits, with greater angular velocities on the inner orbits and smaller ones on the outer orbits (similar to the Solar system).

\subsection{Role in the Formation of the Bulge}

When devouring certain objects, the central supermassive black hole emits lots of entangled pairs of particles in every direction, the members of which do not travel in the same direction, thereby hitting different stars. This can be likened to the black hole shooting around with a double barrel gun whose barrels are not parallel, but are at an angle to one another, and the black hole is aiming at the stars of the galaxy. Obviously, such hits only have a probability of some account within a sphere of a certain radius, and we believe this is what becomes a bulge. The stars within the bulge receive several hits, therefore one star may be entangled to several others, resulting in their chaotically altered orbits. As the resultant force of the attraction forces of the pairs probably points towards the central supermassive black hole, the bulge may contract over time. Albeit with a low probability, it may occur that one particle of an entangled pair hits a star within the bulge, with the other particle of the same pair impacting into one in the halo. In this case, the star in the halo will pick up the angular velocity of the star hit in the bulge, which will probably result in significant acceleration, but will not be detached from the galaxy, due to the attractive force between the entangled particles. (Bosch F., 1998)

\subsection{The Case of Barred Spiral Galaxies}

This type of galaxy may form if a gamma-ray burst occurs in the core (see Figure 1). The gamma-ray burst emits a huge amount of entangled pairs of particles in a thin ray, spraying the stars in its way, which will then attract one another in pairs, forming a chain of stars. It is of course improbable to have 
one particle of an entangled pair go left and the other right; therefore, it is more appropriate to speak of two chains of stars, indicated in different colours in the figure.

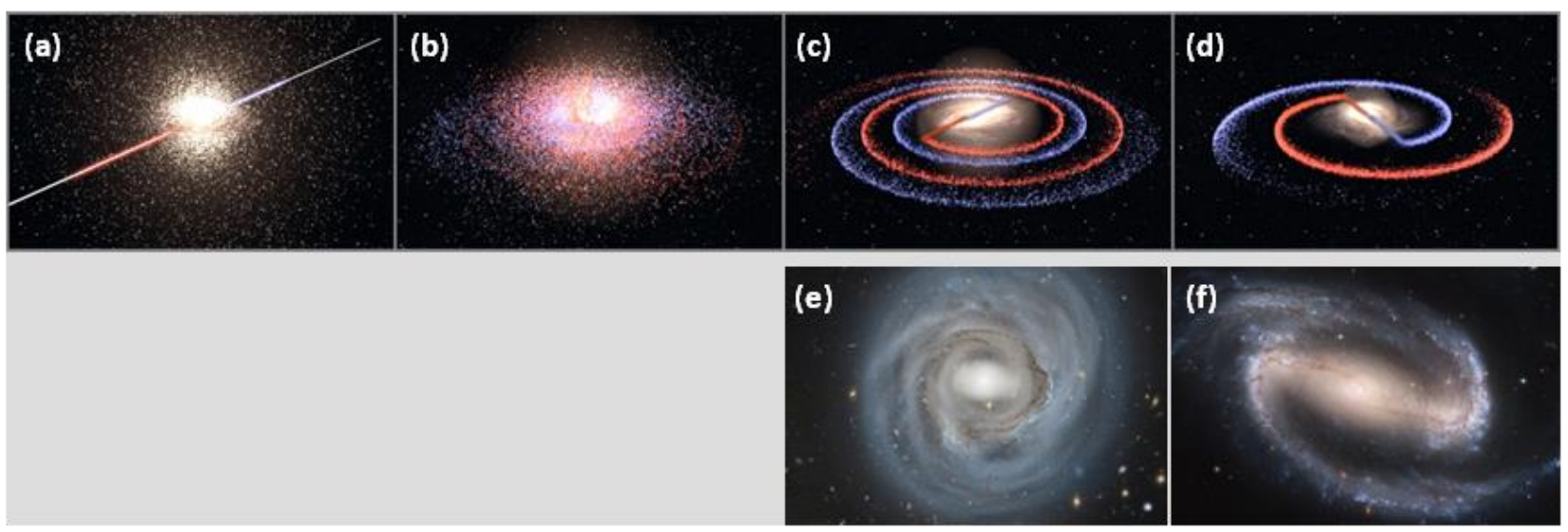

Figure 1. The effect of the attractive force in case of a gamma-ray burst in the core.

The phases of evolution and development of barred spiral galaxies. In figures (a)-(d), we have indicated the stars hit by particles of entangled pairs emitted in 2 different directions in red and blue:

(a) A gamma-ray burst occurs within the core.

(b) In the beginning, the stars hit by the particles seem to follow their original orbits due to their inertia.

(c) The rearrangement becomes visible. The arms are markedly long.

(d) The arms shorten considerably, the bulge contracts slightly, its diameter becoming shorter than the bar.

(e)-(f) Examples of galaxies in the (c) and (d) phases: the NGC 4921 galaxy and the NGC 1300 galaxy (Source of image (e): Astronomy Now https://astronomynow.com/2015/07/27/impact-of-cosmic-wind-on-galaxyevolution-revealed/Source of image (d): NASA https://apod.nasa.gov/apod/ap080622.html)

As the stars move faster on the inner orbits and with smaller angular velocities on the outer orbits, both chains of stars outside the bulge will wind up in the form of a spiral, forming the arms. Initially, densely wound long arms are formed, which, however, shorten over time, we presume this is due to the force of attraction among the stars forming the chain. All intensity peaks of the light curve of the gamma-ray burst (several are possible) result in a new spiral arm branching off, as the object may rotate during the burst. Over time, the angular velocity of the stars forming the chain of stars will become constant, smaller than the average velocity of the stars on the inner orbits, but greater than the stars on the outer orbits. The chains therefore sweep through the entire galaxy, and gravitational forces will pull the other stars of the galaxy into the orbital plane of the chain, resulting in collisions and clusters of stars. This flattens the galaxy out, forming the disc of the galaxy, with only the bulge remaining, as the conditions have already stabilised in there. In the part containing the chain of stars, the bar will again begin to rotate with the arm, but will be straight because the angular velocities of the stars in the bulge are not different due to the earlier impacts. The bar will attract part of the stars in the bulge due to its gravitational attraction, and its length will be equal to the initial diameter of the bulge. Later, however, the bulge will contract and become brighter. Consequently, while the bulge diameter and the bar length are nearly equal in spiral galaxies with densely wound and long arms, the bar is longer in galaxies with short arms. (Abraham R. G. \& Sidney van den Bergh 2001, Courteau S. \& Dutton 2015) The stars hit by the gamma-ray burst are a negligible minority among the total number of stars in the galaxy. We think that the phenomenon of the galaxy clustering the majority of the stars 
into its arms cannot be explained solely by gravitational attraction. Sooner or later, a new gamma-ray burst is likely to hit the galaxy. If this happens within the galaxy, then it is quite improbable that this straight line should be in the plane of the galaxy, and would only hit very few stars as a result (maybe not a single one), causing a negligible effect. On the other hand, if the hit comes from outside, from another galaxy, it will spray the entire galaxy with entangled particle pairs. In case one particle of such an entangled pair hits a star of a chain of stars and the other particle hits another star of the galaxy, this star would eventually become part of the chain, as the members of the chain are interconnected by many millions of pairs, forming a unit, therefore the orbit of this individual star will adapt to that of the others, and not vice versa. This effect, combined with gravitational attraction, clusters the majority of the stars of the galaxy into arms. The stars remaining in the halo have probably not been hit (or just by a few particles) or have remained far from the arms due to the causes mentioned at the description of the bulge.

\subsection{The Case of Unbarred Spiral Galaxies}

This case may happen when the gamma-ray burst does not occur in the central core but somewhere within the galaxy and the distance between the line of the gamma-ray burst and the central supermassive black hole is not "too large". By this we mean that this distance does not exceed the radius of the bulge considerably. (Figure 2)

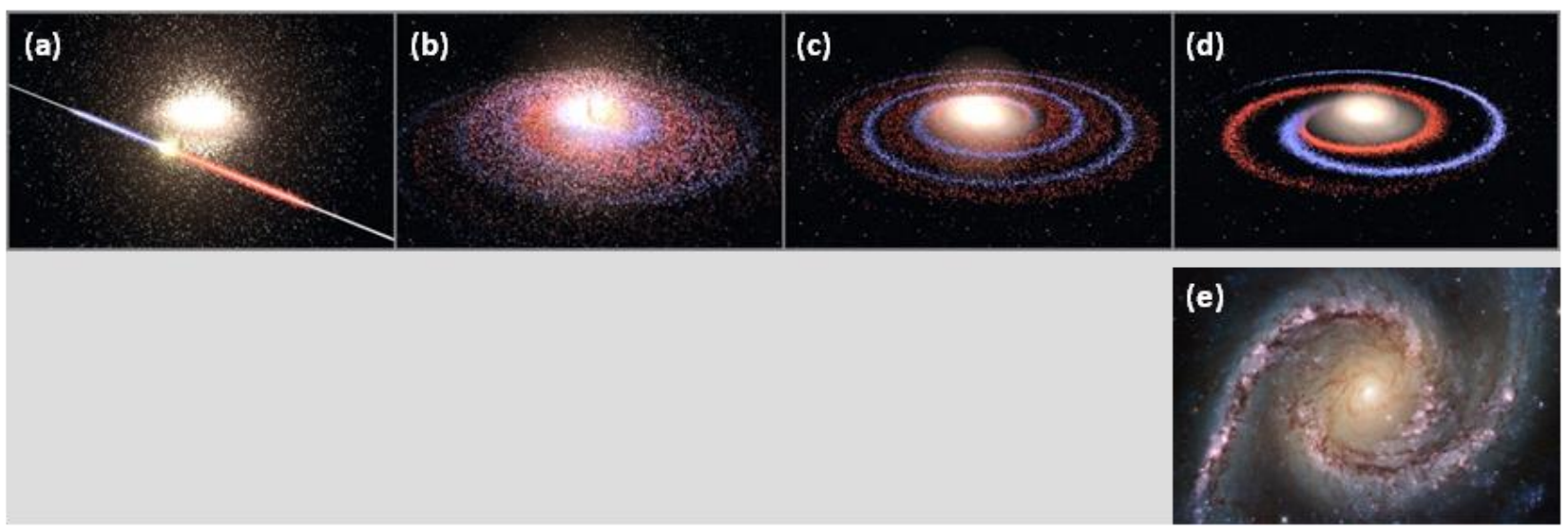

Figure 2: The effect of the attractive force in case of a gamma-ray burst outside the core, near the core. The phases of evolution and development of unbarred spiral galaxies.

(a) A gamma-ray burst occurs near the core.

(b) In the beginning, the stars hit by the particles seem to follow their original orbits due to their inertia.

(c) The rearrangement becomes visible. In this case, the arms stem out from a single point.

(d) The arms shorten considerably.

(e) An example of phase (d) may be the NGC 1566 galaxy.

(Source of image (e): NASA https://www.nasa.gov/content/hubble/grand-swirls-from-nasas-hubble)

In this case, we obtain a spiral as if we grabbed a chain of stars by the star closest to the black hole and wound it up, i.e. there will be no bar and the arms stem out from a single point. Naturally, it is also 
valid here that the intensity peaks of the gamma-ray burst cause the further branching arms to appear. (Brammer G. B. et al. 2012, Burkert, A. et al. 2010)

Our explanation given to the formation of the NGC 4622 (Figure 3) spiral galaxy may be conclusive in supporting this hypothesis, as this explanation is plausible, and it follows from what has been discussed above. Presently there is only one very complicated theory to explain the rotation of the arms in opposite directions. (Tosa M. 1994)

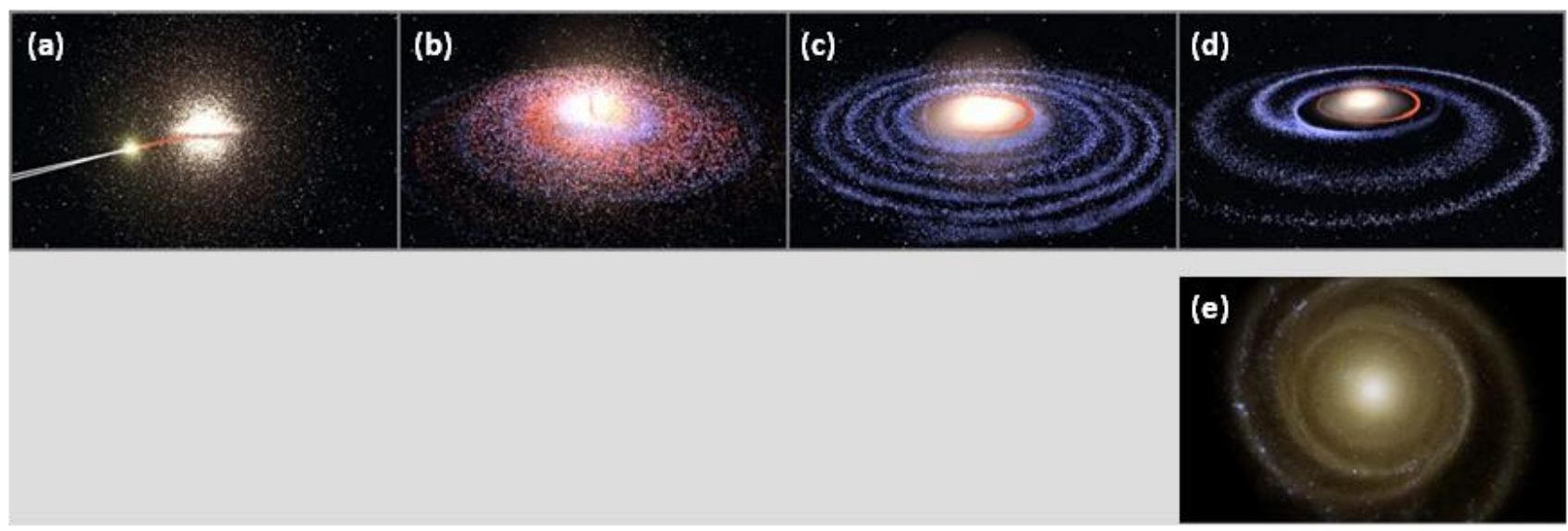

Figure 3: The gamma-ray burst occurs close to the core with two intensity peaks, also hitting a star of the core.

(a) Gamma-ray burst with two intensity peaks.

(b) The rearrangement begins

(c) The three arms become visible; two outer (blue) and an inner (red) arm. The two outer arms rotate counterclockwise, and the inner arm in the opposite direction.

(d) The arms shorten. (e) An example of phase (d) is NGC 4622.

(Source of image (e): Hubble https://www.spacetelescope.org/images/opo0203a/)

As it can be seen from the image, the inner arm rotates clockwise and the two outer arms rotate in the opposite direction. We believe the gamma-ray burst occurred at the meeting point of the inner and outer arms with two intensity peaks, hence the two outer arms. As there is only one inner arm, it is possible that a "nearby” star in the core has been hit. The opposite direction of rotation may have evolved by the majority of the stars forming the inner arm defining a clockwise direction (i.e. the resultant of the momenta pointed in that direction, probably coinciding with the direction of rotation of the star that was hit), while the majority of the stars defining the two outer arms indicated an opposite direction. 


\subsection{The Formation of Ring Galaxies and Certain Dwarf Galaxies}

If the gamma-ray burst takes place somewhere within the galaxy, but not in the core, and the distance mentioned above is "too large", we think that the chain of stars is either formed into a ring or detaches from the galaxy (Figure 4).

It may take up the shape of a ring, as the differences in angular velocity are not significant and there is no star on an inner orbit among the stars forming the chain, almost at an identical distance from the central supermassive black hole, causing the two-star chains to wind nearly along the same arc. The gamma-ray burst does not stop at the boundary of the galaxy, and is most likely to hit other galaxies as well. Therefore, the stars of the chain and the outer galaxies will attract each other in pairs, and if this attractive force is greater than the gravitational force of the galaxy, the chain will be detached. Before that, however, it will sweep along the outer part of the galaxy. After the chain has moved away from the galaxy, its stars fuse together in a supermassive black hole, as the gravitation of the galaxy no longer has an effect on them, only the attraction by pairs, which concentrates the stars in a singular point. The mass of the resulting black hole may be several billion times the mass of the Sun. The remaining galaxy will be a dwarf galaxy, which may be of any type.

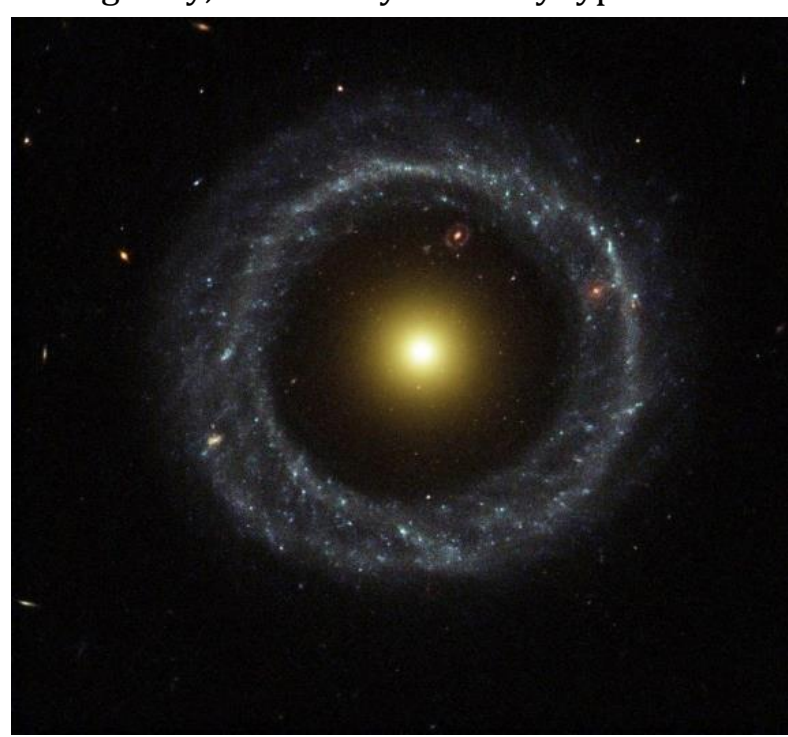

Figure 4: In case of a gamma-ray burst distant from the core, the galaxy will be of a ring type. The figure shows an example of such a galaxy (Source of image: NASA https://www.nasa.gov/multimedia/imagegallery/image_feature_1747.html)

\subsection{Explanation of the Formation of Flocculent Galaxies}

A gamma-ray burst whose light curve contained many intensity peaks, each defining a little arm, may have occurred in the galaxy (Figure 5). (David L., et al. 1996) 


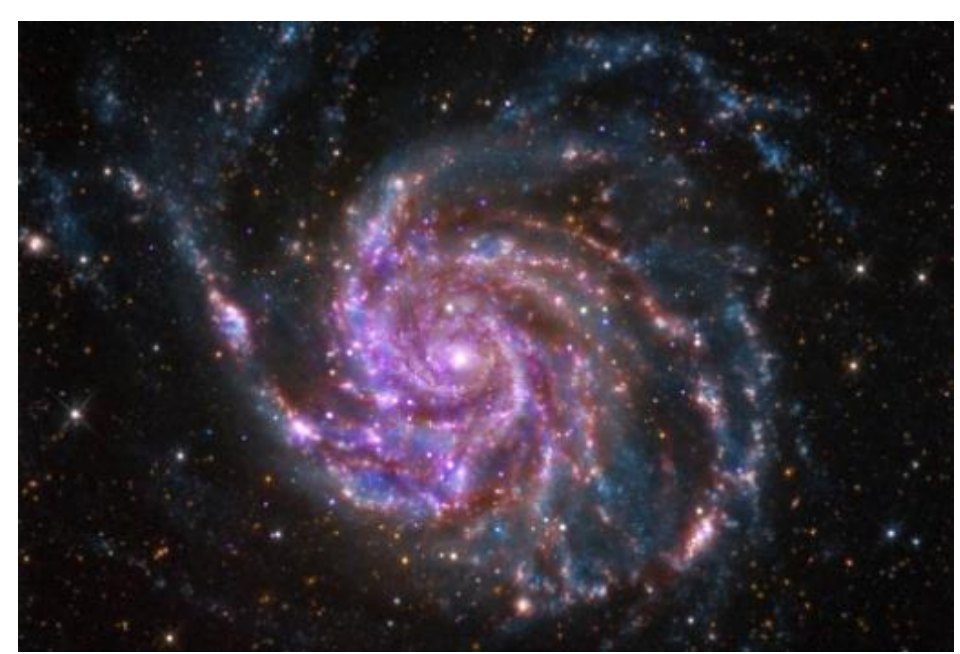

Figure 5: The gamma-ray burst had many intensity peaks, which resulted in a flocculent galaxy. An example of one such galaxy is M101

(Source of image: NASA https://www.nasa.gov/mission_pages/chandra/multimedia/spiral-galaxy-m101.html)

\subsection{The Case of the Elliptical Galaxy}

If the gamma-ray burst occurs in an external galaxy, hitting the galaxy examined, this will spray the entire galaxy with large quantities of entangled particle pairs - or some event producing the same result may happen within the galaxy (Figure 6).

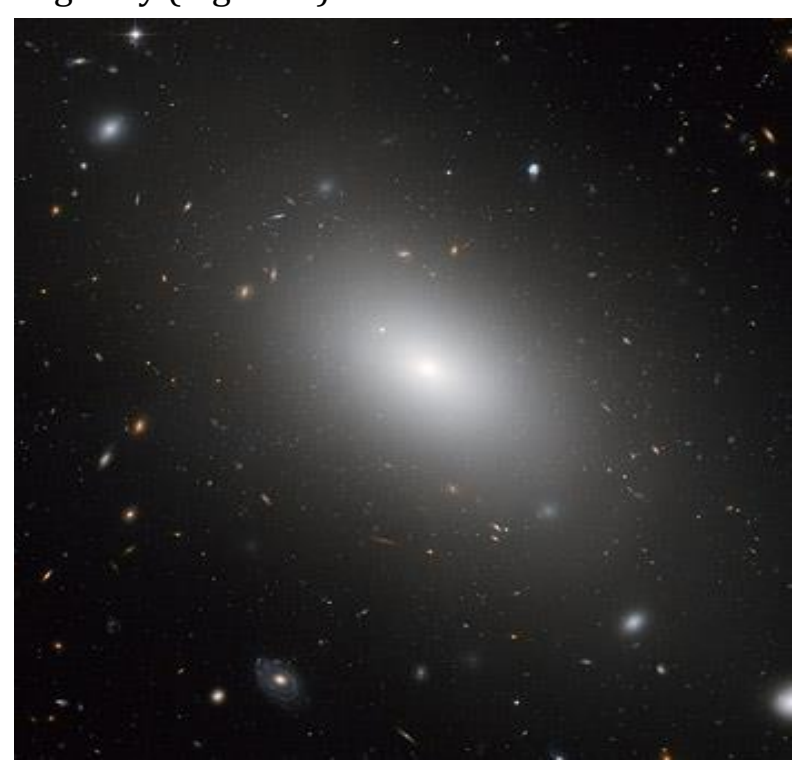

Figure 6: The gamma-ray burst comes from an external galaxy, resulting in an elliptical galaxy. One example of this is NG 1132

(Source of image: HubbleESA https://www.spacetelescope.org/images/heic0804a/)

Due to the large distance of the galaxies, the radius of the gamma-ray burst will almost certainly broaden to include the entire galaxy, not just part of it. The stars will begin to attract one another by pairs, consequently getting packed closer together, but retaining their original spherical or ellipsoid shapes. Due to the attractive forces acting in every direction, the stars will not move in the same direction around the core, their orbits will become erratic. As a whole, it resembles the shape and 
structure of the bulge. Less collisions will occur, and the rate of star formation is also lower than in spiral galaxies. (Fukugita M. et al. 1996, Capaccioli M. 1987) If a new gamma-ray burst occurs in the galaxy, then it will have no significant effect on the shape of the galaxy, as the angular velocities do not vary with the distance from the core.

\section{The effect of the presumed attractive force on the structure of the universe}

If we only look at the sky with our telescopes in a narrow angle, we can still see multitudes of galaxies. Similarly, the ray of a gamma-ray burst will hit many galaxies, which, according to our hypothesis, will attract one another, and therefore will be arranged into the line of this ray.

Examining the effect of the gamma-ray burst occurring in the spiral galaxy of Figure 8, we will find that the galaxies hit in the direction of the blue arrow will attract the stars forming the blue spiral arm and thereby the entire galaxy. This force is indicated by $\boldsymbol{F}_{\boldsymbol{b}}$. Similarly, another force, indicated by $\boldsymbol{F}_{\boldsymbol{a}}$, is obtained in the red direction. It is highly improbable that these two forces should be identical, therefore the galaxy will accelerate in the direction of the greater force. Observing this from our galaxy, we will only see the galaxy approaching us if it accelerates in the blue direction and even then only along its section indicated in yellow; in all other cases, we see it accelerating away from us. If only gravitational attraction was acting on the galaxies, their collisions would be highly improbable, considering the enormous distances. We think that because of this, the generally accepted assumption that the Milky Way will collide with the Andromeda galaxy, also corroborates our hypothesis.

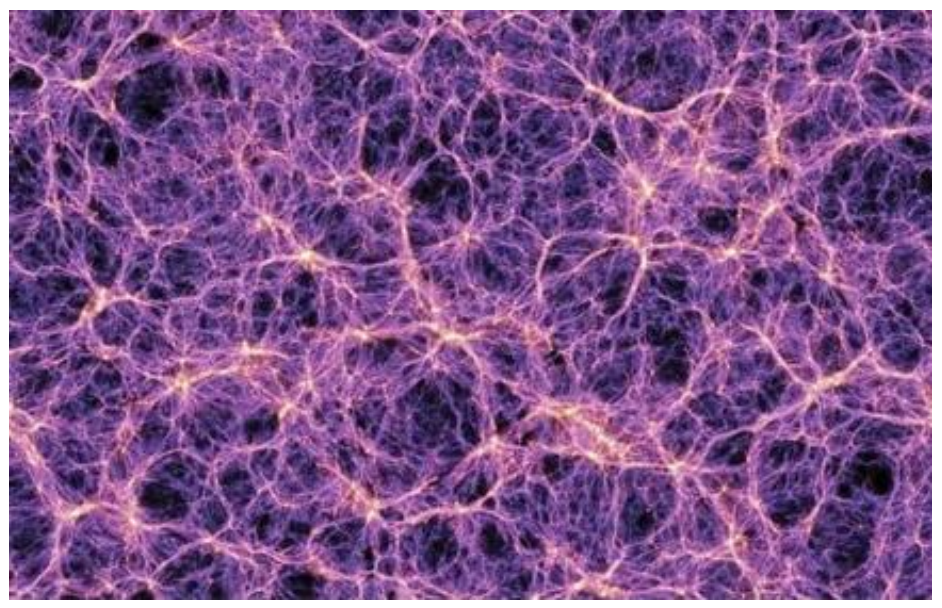

Figure 7: According to our assumption, it shows the structure of groups of galaxies arranged by gamma-ray bursts that occurred long ago.

(Source of image: Science http://www.sciencemag.org/news/2010/07/new-way-map-universe) 


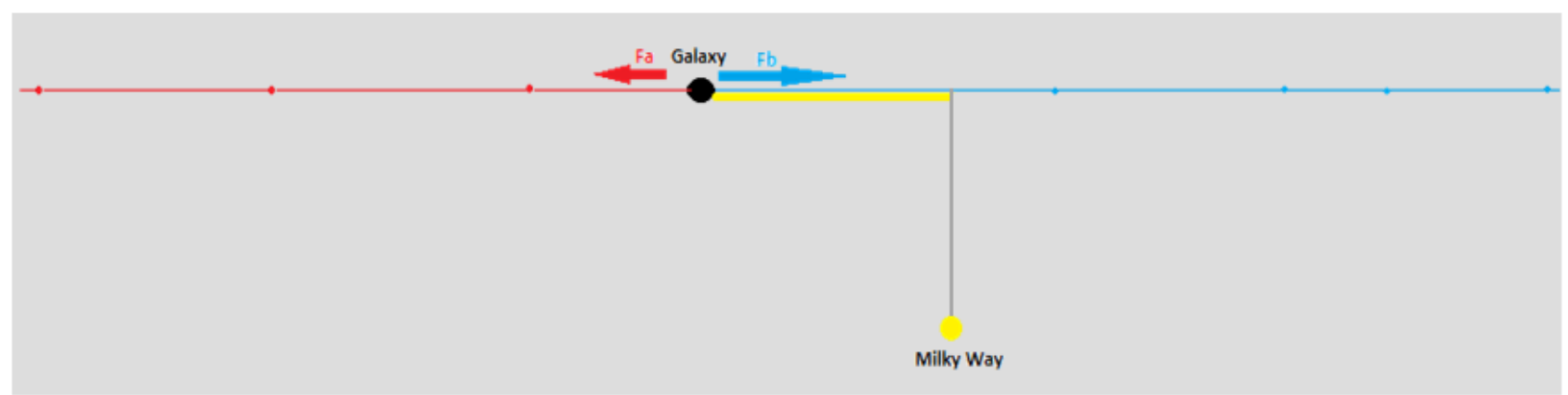

Figure 8: Movement of galaxies - They only seem to be approaching us in the yellow section; in every other case, the galaxies are moving away from us with increasing speed

\section{CONCLUSION}

Gyula Dávid, physicist and astronomer, once said during a lecture that "the laboratory of the poor physicist is the starry sky". Well, we too have looked up to the sky, and we observed, starting from the assumption that the entangled pairs of particles attract each other independently from the distance between them, and that gamma-ray bursts can separate these particles in a very large number and shoot them in a jet, and we may end up with a theory universal in the sense that it provides a universal explanation of the shapes of the various galaxies, the spiral arms, the bar, the bulge, the arms of the galaxy NGC 4622 rotating in opposite directions, the single stars orbiting very quickly at the edges of galaxies, the formation of black holes of masses billions of times heavier than the Sun, the spider weblike structure of the universe, etc., contrary to contemporary theories that explain these on separate foundations, if they explain them at all.

\section{References}

[1] R. G. Abraham - S. van den Bergh (2001) The Morphological Evolution of Galaxies. Science, 293, 5533, 1273-1278,

[2] D. L. Block - R. J. Wainscoat (1991) Morphological differences between optical and infrared images of the spiral galaxy NGC309. Nature 353, 48-50

[3] F. Bosch (1998) The Formation of Disk-Bulge-Halo Systems and the Origin of the Hubble Sequence. Astrophys. J., 507:601 614

[4] G. B. Brammer et al. (2012) 3D-HST: a wide-field grism spectroscopic survey with the Hubble Space Telescope. Astrophys. J. Suppl. Ser.200, 13

[5] A. Burkert et al. (2010) High-redshift star-forming galaxies: angular momentum and baryon fraction, turbulent pressure effects, and the origin of turbulence. Astrophys. J. 725, 23242332

[6] J. 0. Burns (1986) Very Large Structures in the Universe. Scientific American 255, 38-47

[7] M. Capaccioli (1987) in IAU Symp. 127, Structure and Dynamics of Elliptical Galaxies, ed. T. de Zeeuw (Dordrecht: Reidel), 47 
[8] E. Costa et al. (1997) Discovery of an X-ray afterglow associated with the $\gamma$-ray burst of 28 February 1997. Nature 387, 783-785

[9] S. Courteau - Dutton (2015) On the global mass distribution in disk galaxies. Astrophys. J. 801, L20

[10] D. L., Block - B. G. Elmegreen - R. J. Wainscoat et al. (1996) Smooth dark spiral arms in the flocculent galaxy NGC2841. Nature 381, 674-676

[11] J. Einasto (1965) Kinematics and dynamics of stellar systems. Trudy Inst. Astroz. Alma-Ata 5,87

[12] A. Einstein - B. Podolsky - N. Rosen et al. (1935) Can quantum-mechanical description of physical reality be considered complete? Phys. Rev. 47, 777-780

[13] A. Einstein (1915) Erklärung der Perihelbewegung des Merkur aus der allgemeinen Relativitätstheorie. Preuss. Akad. Wiss. Berlin, 47, 831-839

[14] M. Fukugita et al. (1996) Peebles The history of the galaxies. Nature 381, 489-495

[15] A. Grant et al. (2015) Entanglement: Gravity's long-distance connection: Wormhole links between black holes could broker quantum-general relativity merger. Science News 188(8): 28-31

[16] R. Horodecki - P. Horodecki - M. Horodecki - K. Horodecki et al. (2009) Quantum entanglement. Rev. Mod. Phys. 81, 865-942

[17] J. Maldacena - L. Susskind, et al. (2013) Cool horizons for entangled black holes Fortsch. Phys. 61, 781

[18] P. Mészáros - M. J. Rees et al. (1997) Optical and long wavelength afterglow from gammaray bursts. Astrophys. J. 476, 232-237

[19] O. Müller - M. S. Pawlowski - H. Jerjen - F. Lelli et al. (2018) A whirling plane of satellite galaxies around Centaurus. A challenges cold dark matter cosmology. Science, 359, 6375, 534-537

[20] M. Tosa (1994) Formation of a retrograde spiral arm induced by a Ram pressure. Astrophys. J. $426 / 2,81-83$

[21] G. Toth - J. P. Ostriker et al (1992) Galactic disks, infall, and the global value of Omega. Astrophysical Journal 389(10) 